03

\title{
Расчет межфазного массообмена в факеле распыла форсунки с учетом кризиса
}

\author{
(C) Н.Н. Симаков \\ Ярославский государственный технический университет, \\ 150023 Ярославль, Россия \\ e-mail: nik_simakov@mail.ru
}

Поступило в Редакцию 16 ноября 2017 г.

В окончательной редакции 29 мая 2019 г.

Принято к публикации 21 октября 2019 г.

Описаны численная модель и результаты расчета межфазного массообмена в двухфазном потоке, образующемся при распыливании жидкости в газе с помощью форсунки. Основу предлагаемой математической модели составляли дифференциальные уравнения нестационарного течения сжимаемой среды, дополненные уравнением массопередачи от газа к каплям. При переходе к разностным аналогам уравнений неразрывности и движения фаз использована известная явная схема Лакса-Вендроффа. Рассчитаны и представлены аксиальные профили скоростей капель, газа и концентраций газовой примеси в свободном факеле распыла, а также радиальные профили концентраций примеси в двухфазном потоке через цилиндрический аппарат с учетом раннего кризиса сопротивления капель и кризиса массообмена фаз, а также особенностей турбулентного трения в газе, обнаруженных в предшествующих экспериментах. Расчетом установлены зависимости расхода газа, концентрации примеси газа на выходе аппарата и количества поглощенной жидкостью примеси от высоты и площади сечения аппарата.

Ключевые слова: форсунка, факел распыла, межфазный массообмен, расчет.

DOI: 10.21883/JTF.2020.04.49077.2566

\section{Введение. Особенности гидродинамики факела распыла}

Для интенсификации тепломассобменных процессов, таких, как сжигание жидких топлив, сушка и гранулирование полимеров, мокрая очистка воздуха от пыли и вредных газовых примесей путем увеличения межфазной поверхности используют распыливание жидкости в газе, например, с помощью форсунок.

Основу расчета таких процессов составляют знания о гидродинамической структуре образующегося факела распыла, силах взаимодействия капель с газом, а также представления об элементарных актах переноса тепла и/или массы на уровне отдельных капель. До сих пор методы удовлетворительного расчета распылительных процессов не разработаны, чем и обусловлена актуальность настоящей работы.

Обычно процесс межфазного массообмена сопровождается параллельно и одновременно протекающим теплообменом, что осложняет их моделирование и расчет. В технологии мокрой газоочистки воздуха от вредных газовых примесей [1], например от оксида серы $\mathrm{SO}_{2}$, процесс межфазного массообмена можно рассматривать без учета теплообмена. Моделирование такого рода массообменных процессов, связанных с абсорбцией газовой примеси (в воздухе) каплями распыленной жидкости (воды), явилось целью настоящей работы.

Для математического моделирования двухфазных потоков известны два основных подхода: метод взаимопроникающих континуумов [2] и теория турбулентных струй [3]. В первом из них каждая из фаз рассматривается как непрерывно распределенная по пространству с переменной (средней по малому объему) плотностью, а скорости фаз полагаются различными. Во втором предполагается, что концентрация дисперсной фазы мала, скорости фаз примерно одинаковы, но при этом учитывается турбулентность течения газовой фазы. Каждый из этих подходов, взятый отдельно, не учитывает описанных ниже важных особенностей факела распыла.

Экспериментально установлено, что течение газа в факеле распыла является турбулентной струей $[4,5]$. Она возникает у корня факела из-за взаимодействия фаз, и с удалением от форсунки развивается как бы независимо от капельного потока, но отличается от однофазного потока структурой и характером турбулентного трения. В частности, безразмерные профили аксиальной скорости газа оказываются более пологими, чем в однофазной струе. Установлено различие скоростей фаз и наличие перепадов давления газа порядка 1-10 Ра по радиусу и оси факела.

Обнаружена также существенная особенность во взаимодействии фаз, названная ранним кризисом сопротивления [4], которая также требует учета при расчете факела распыла и заключается в следующем.

При распыливании жидкости форсункой образуются капли со средним диаметром $d$ порядка $10^{-4} \mathrm{~m}$. При таких размерах и существенном различии динамических коэффициентов вязкости жидкости капель и обтекающего их газа (для воды и воздуха примерно в 60 раз) можно 


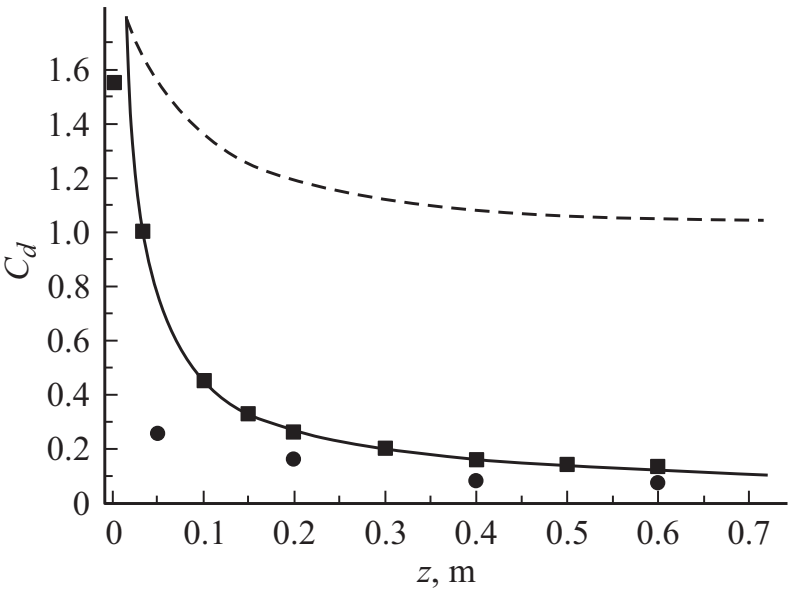

Pис. 1. Зависимость коэффициента сопротивления $C_{d}$ капель от расстояния $z$ до форсунки: - - данные эксперимента [4] для капель воды на оси факела форсунки при давлении воды на ней $P_{l}=5 \cdot 10^{5} \mathrm{~Pa}$; • - то же для капель на границе факела; сплошная кривая - аппроксимация данных эксперимента на оси факела по формуле (13); штриховая кривая - расчет по формуле (3) с использованием экспериментальных данных [4] для скоростей газа и капель, их размеров.

пренебречь деформацией капель и внутренним течением жидкости в них, рассматривая их как твердые шарики.

Гидродинамическую силу сопротивления капли при ее обтекании газом обычно вычисляют по формуле

$$
F=C_{d} S \rho V^{2} / 2,
$$

зная относительную скорость $V$ капли в газе и коэффициент $C_{d}$ гидродинамического сопротивления. Здесь и ниже обозначено: $S=\pi d^{2} / 4-$ площадь центрального сечения сферической капли, $\rho$ - плотность газа, $\mu-$ динамический коэффициент вязкости газа.

Для обтекания шара ламинарным потоком при малых числах Рейнольдса $\operatorname{Re}=V d \rho / \mu \ll 1$ известна формула Стокса

$$
C_{d}=24 / \operatorname{Re} .
$$

A для обтекания в переходном $2<\operatorname{Re}<700$ известна зависимость Клячко

$$
C_{d}=24 / \operatorname{Re}+4 / \operatorname{Re}^{1 / 3},
$$

которая в указанном диапазоне хорошо аппроксимирует экспериментальные данные, обобщенные кривой Рэлея [6,7].

В работах $[4,5]$ результатами эксперимента показано (рис. 1), что в сильно турбулентном потоке при $\mathrm{Re} \approx 100$ величина $C_{d}$ для капель может уменьшаться в 4-7 раз по сравнению с определяемыми формулой (3) общеизвестными значениями. Такой же ранний кризис сопротивления наблюдался на одиночном твердом шарике, обтекаемом газовой струей в конфузоре [8].

Заметим, что приведенные на рис. 1 экспериментальные точки при $z>0.15 \mathrm{~m}$ близки к значениям, соответствующим формуле Стокса (2).
В качестве одной из причин, обусловливающих ранний кризис сопротивления сферической частицы, рассматривалась гипотеза о влиянии сильной турбулентности газового потока, которую конфузор еще больше усиливал по сравнению со свободной струей и делал достаточной для возникновения раннего кризиса на одиночном твердом шарике [8].

Это предположение было подтверждено численным моделированием обтекания шара свободным газовым потоком как ламинарным, так и сильно турбулентным [9].

Из вышесказанного был сделан вывод: при математическом и численном моделировании факела распыла как двухфазного потока с учетом всех его особенностей для описания движения обеих фаз единым образом целесообразно использовать сочетание двух известных подходов: метода взаимопроникающих континуумов [2] и теории турбулентных струй [3]. Эта идея недавно использовалась в работе [10] для расчета гидродинамики как свободного факела распыла, так и потока, ограниченного стенками цилиндрического аппарата.

\section{Моделирование массообмена в свободном факеле распыла}

Уравнения гидродинамики двухфазного потока в факеле распыла форсунки, составляющие основу рассматриваемой модели, подробно представлены в работе [10], но ради целостности изложения темы и удобства читателей настоящей работы приведем их здесь снова.

Турбулентное течение газовой фазы описывают в цилиндрической системе координат нестационарным уравнением неразрывности

$$
\frac{\partial \rho}{\partial t}+\frac{\partial\left(\rho w_{z}\right)}{\partial z}+\frac{1}{\rho} \frac{\partial\left(r \rho w_{r}\right)}{\partial r}=0
$$

и уравнениями движения для осредненных по времени аксиальной $w_{z}=w_{z}(r, z)$ и радиальной $w_{r}=w_{r}(r, z)$ компонент скорости газа:

$$
\begin{gathered}
\frac{\partial w_{z}}{\partial t}+w_{z} \frac{\partial w_{z}}{\partial z}+w_{r} \frac{\partial w_{z}}{\partial r}=\frac{1}{\rho r} \frac{\partial(r \tau)}{\partial r}-\frac{1}{\rho} \frac{\partial P}{\partial z}+\frac{f_{z}}{\rho} \\
\frac{\partial w_{r}}{\partial t}+w_{z} \frac{\partial w_{r}}{\partial z}+w_{r} \frac{\partial w_{r}}{\partial r}=-\frac{1}{\rho} \frac{\partial P}{\partial r}+\frac{f_{r}}{\rho}
\end{gathered}
$$

Для жидкой фазы (индекс ,l“ $)$ используются аналогичные уравнения:

$$
\begin{aligned}
& \frac{\partial \alpha}{\partial t}+\frac{\partial\left(\alpha u_{z}\right)}{\partial z}+\frac{1}{r} \frac{\partial\left(r \alpha u_{r}\right)}{\partial r}=0, \\
& \frac{\partial u_{z}}{\partial t}+u_{z} \frac{\partial u_{z}}{\partial z}+u_{r} \frac{\partial u_{z}}{\partial r}=-\frac{f_{z}}{\alpha \rho_{l}}, \\
& \frac{\partial u_{r}}{\partial t}+u_{z} \frac{\partial u_{r}}{\partial z}+u_{r} \frac{\partial u_{r}}{\partial r}=-\frac{f_{r}}{\alpha \rho_{l}} .
\end{aligned}
$$

Здесь $P$ и $\rho$ - давление и плотность газа, $\tau-$ напряжение турбулентного трения внутри газа, $f_{r}$ и $f_{z}$ - объемные плотности сил межфазного взаимодействия, $\alpha-$ 
объемная доля жидкости, $\rho_{l}-$ физическая плотность капель, $u_{z}, u_{r}$ - аксиальная и радиальная компоненты скорости жидкости.

Система уравнений (4)-(9) не замкнута, так как в ней 9 неизвестных функций: $w_{z}, w_{r}, \rho, P, \alpha, u_{z}, u_{r}, f_{r}, f_{z}$. Замыкание системы выполним следующим образом.

Во-первых, можно предположить, что течение газа в факеле распыла адиабатное, и воспользоваться уравнением Пуассона

$$
\frac{P}{\rho^{\gamma}}=\text { const }=\frac{P_{0}}{\rho_{0}^{\gamma}},
$$

из которого следуют соотношения

$$
d P=\gamma \frac{P}{\rho} d \rho=w_{s}^{2} d \rho=w_{s 0}^{2}\left(\frac{\rho}{\rho_{0}}\right)^{\gamma-1} d \rho,
$$

где $w_{s}=(\gamma P / \rho)^{1 / 2}-$ скорость звука в газе, $\gamma-$ постоянная адиабаты, индекс „,““ обозначает начальные значения величин.

Подстановкой последнего выражения из (10) в (5) и (6) можно исключить давление $P$ газа из системы уравнений (4)-(9).

Во-вторых, для напряжения $\tau$ турбулентного трения газа в работе [5] на основании экспериментальных данных получена зависимость

$$
\tau=-\rho \frac{r Z}{2 \xi^{2}}\left(\frac{\partial w_{z}}{\partial r}\right)^{2},
$$

где $Z=z+Z_{0}$ - осевая координата, отсчитываемая от полюса газовой струи, $z$ - то же от форсунки, $Z_{0}-$ расстояние от полюса до форсунки, $\xi=\operatorname{const}\left(P_{l}\right)^{1 / 2}$. При избыточном давлении воды на форсунке $P_{l}=5 \cdot 10^{5} \mathrm{~Pa}$ были получены значения $Z_{0}=390 \mathrm{~mm}, \xi=11.9$.

Заметим, что представление (11) отличается от аналогичного для однофазной турбулентной газовой струи по „новой“ гипотезе Прандтля

$$
\tau=\rho l^{2}\left(\frac{\partial w_{z}}{\partial r}\right)^{2}=\rho v_{t} \frac{\partial w_{z}}{\partial r},
$$

где $l$ - путь смешения, кинематический коэффициент турбулентной вязкости $v_{t}=$ const - эмпирическая постоянная [11].

При подстановке выражения (11) в уравнение (5) для упрощения последнего зависимостью $\rho$ от $r$ в дозвуковом течении допустимо пренебречь.

В-третьих, действующую со стороны газа на отдельную каплю силу сопротивления обычно выражают формулой (1), где $\mathbf{V}=\mathbf{u}-\mathbf{w}-$ относительная скорость движения капли в газе. Тогда для компонент объемной плотности сил межфазного взаимодействия запишем

$$
f_{z, r}=F_{z, r} n=\frac{F_{z, r} \alpha \rho_{l}}{m_{d}}
$$

где $n$ - счетная концентрация капель, $m_{d}=V_{d} \rho_{l}-$ масса, $V_{d}$ - объем капли.
Выше отмечалось, что значения коэффициента сопротивления $C_{d}$ капель в факеле распыла и его зависимость от числа Рейнольдса $\operatorname{Re}=\rho d V / \mu$ носят аномальный характер $[4,5]$. У „корня“ факела, вблизи форсунки, значения $C_{d}$ приблизительно соответствуют известным из литературы зависимостям, например, формуле Клячко (3), и резко уменьшаются в 4-7 раз при удалении от форсунки (рис. 1).

Из рис. 1 очевидно: уменьшение $C_{d}$ с увеличением расстояния $z$ от форсунки по оси факела удовлетворительно аппроксимируется зависимостью

$$
C_{d}(0, z)=0.45(z / 0.1)^{-3 / 4} .
$$

Еще очевидно, что на „границе $r=r_{\lim }(z)=z \operatorname{tg} \varphi$, определяемой наиболее удаленными от оси траекториями капель, образующими с ней угол $\varphi=32.5^{\circ}$, равный половине корневого угла факела, значения $C_{d}\left(r_{\mathrm{lim}}, z\right)$ примерно вдвое (в 1.8 раза) меньше, чем $C_{d}(0, z)$ на оси факела, что в последующих расчетах также учтено по формуле

$$
C_{d}(r, z)=C_{d}(0, z)\left(0.45 \exp \left(-6 r / r_{\lim }(z)\right)+0.55\right) .
$$

Для расчета массообмена фаз (без учета их теплообмена) система уравнений (4)-(14) должна быть дополнена соотношениями, учитывающими конвективный перенос примесного газового компонента в потоке и его массопередачу от газа к каплям жидкости.

С учетом аналогии явлений межфазного тепло- и массообмена [11] для плотности потока массы примеси от газа к отдельной капле мы можем написать уравнение

$$
j=k\left(C-C_{l}\right) \rho,
$$

аналогичное уравнению Ньютона-Рихмана для потока тепла. Также по аналогии с коэффициентом теплоотдачи можно определить коэффициент массоотдачи

$$
k=\mathrm{Nu}_{D} D / d,
$$

а диффузионное число Нуссельта определить предложенной для теплообмена формулой Ранца-Маршалла

$$
\mathrm{Nu}_{D}=2+0.6 \operatorname{Re}^{1 / 2} \operatorname{Pr}_{D}^{1 / 3} \text {. }
$$

Здесь и далее по тексту: $C=C(r, z)-$ концентрация газовой примеси в воздухе вдали от капли, а $C_{l}-$ то же вблизи ее поверхности, $D-$ коэффициент диффузии примеси (для $\mathrm{SO}_{2}$ в воздухе $D=2.45 \cdot 10^{-5} \mathrm{~m}^{2} / \mathrm{s}$ ), $\operatorname{Pr}_{D}=\mu /(\rho D)=0.637$ - диффузионное число Прандтля (иногда называемое числом Шмидта $\mathrm{Sc}$ ).

Без учета кризиса массообмена, аналогичного кризису теплообмена [12], формула (17) дает значения $\mathrm{Nu}_{D}>2$. Для учета кризиса массообмена нужно использовать значение $\mathrm{Nu}_{D}=2$.

Для упрощения модели концентрацию, легко абсорбируемой (как при хемосорбции) каплями жидкости 
газовой примеси у их поверхности, можно принять $C_{l}=0$. Это - внешняя задача массообмена фаз.

Тогда поток массы примеси от газа ко всем каплям в единице объема факела с учетом (15) можно представить в виде

$$
J=\frac{j 4 S \alpha}{V_{d}}=\frac{6 j \alpha}{d}=\frac{6 k \rho \alpha C}{d} .
$$

Теперь уравнение конвективно-диффузионного переноса примесного компонента в газовом потоке и его массопередачу от газа к каплям жидкости можем записать в виде

$$
\frac{\partial C}{\partial t}+w_{z} \frac{\partial C}{\partial z}+w_{r} \frac{\partial C}{\partial r}=D \Delta C-\frac{J}{\rho} .
$$

Уравнение (19) совместно с соотношениями (16)-(18) дополняет систему уравнений (4)-(14) и позволяет рассчитывать изменение концентрации газовой примеси $C=C(r, z)$ в двухфазном потоке факела распыла форсунки как с учетом кризиса сопротивления капель и кризиса массообмена фаз, так и без учета этих кризисов.

В численной модели факела распыла перед тем, как представить дифференциальные уравнения их разностными аналогами, целесообразно перейти к безразмерным переменным, поделив значения координат $r$ и $z$ на начальный (минимальный) радиус $r_{0}=z_{0} \operatorname{tg} \varphi$ факела в расчетной области $\left(z_{0}=100 \mathrm{~mm}\right.$ от выходного отверстия форсунки), скоростей $w, u, V$ и $w_{s}$ - на начальную скорость капель (струи жидкости) $u_{0}, \rho-$ на плотность $\rho_{0}$ покоящегося газа вдали от факела, $t-$ на $t_{0}=r_{0} / u_{0}$, a $C(r, z)$ - на начальную концентрацию $C_{0}$ примеси в неочищенном газовом потоке. Внешний вид уравнений (4)-(9) при этом не изменится, а у слагаемых в правых частях уравнений появятся соответствующие дополнительные коэффициенты.

Как и в работах $[5,10,12]$, при переходе от дифференциальных уравнений (4)-(9), (19) к их разностным аналогам с учетом представлений (1), (10)-(18) на прямоугольной пространственной сетке $(i, j)$, для аппроксимации конвективных членов использовалась явная двухшаговая разностная схема Лакса-Вендроффа [13]. В этой схеме для каждого временного слоя на первом, вспомогательном, шаге вычислений находим промежуточные значения зависимых переменных при $t=(n+1 / 2) \Delta t$ по схеме Лакса с половинным шагом $\Delta t / 2$ по времени. Значения величин на предыдущем слое при этом усредняем по четырем ближайшим узлам. На втором, основном, шаге вычислений с полным шагом $\Delta t$ по времени в выражениях, аппроксимирующих пространственные производные, используем найденные промежуточные значения величин и находим новые значения переменных. Затем цикл повторяем.

Схема Лакса-Вендроффа центрирована по времени [13], благодаря чему численные эффекты вязкости и диффузии в ней значительно меньше, чем в одношаговой схеме Лакса, что дает более близкие к истинным профили скоростей каждой из фаз.

Для устойчивости разностной схемы Лакса-Вендроффа необходимо выполнить условие Куранта-Фридрихса-Леви [13] при равных пространственных шагах сетки $\Delta z=\Delta r$ имеющее вид

$$
\Delta t \leq \frac{\Delta z}{\sqrt{2\left(w_{s}^{2}+w_{z}^{2}+w_{r}^{2}\right)}} .
$$

В сочетании со схемой Лакса-Вендроффа аппроксимация диффузионного члена в уравнении (19) проводилась по явной схеме первого порядка точности [13], для которой в случае двумерной сетки условие устойчивости имеет вид

$$
\Delta t \leq \frac{(\Delta z)^{2}}{4 D} .
$$

Для обеспечения устойчивости разностной схемы в целом необходимо одновременное выполнение обоих условий (20) и (21) [13], из которых первое в данном случае оказалось более сильным.

Преимуществом предложенной модели является то, что ее разностные уравнения позволяют вычислять все переменные по простой явной схеме.

Одна из трудностей построения численной модели заключается в задании подходящих граничных условий, сохраняющих устойчивость разностной схемы. В отличие от внутренних точек в граничных узлах расчетной области разностная схема с неизбежностью имеет иной вид, так как для аппроксимации пространственных производных здесь используются не двухсторонние, а односторонние разности. Кроме того, нужно учесть, что на оси симметрии (при $r=i \Delta r=0$ ) радиальные скорости фаз $w_{r}=u_{r}=0$, производные по $r$ от некоторых переменных также могут обращаться в нуль.

На верхней (входной) границе расчетной области $(j=0)$ с учетом экспериментальных данных нужно задать профиль объемной доли жидкости, например, треугольной формы $\alpha\left(r, z_{0}\right)=3\left(r_{n} / r_{0}\right)^{2}\left(1-r / r_{0}\right), r_{n}$ радиус выходного отверстия форсунки, $r_{0}=z_{0} \operatorname{tg} \varphi$.

На верхней границе нужно задать также радиальные профили компонент скорости жидкости $u_{z}\left(r, z_{0}\right)$ и $u_{r}\left(r, z_{0}\right)$. Первый из них может быть прямоугольным, трапециевидным или более сложным, второй с учетом характера истечения жидкости из сопла форсунки стоит задать функцией радиуса такого вида: $u_{r}\left(r, z_{0}\right)=u_{z}\left(r, z_{0}\right) r / z_{0}$.

При расчете свободного факела на боковой (внешней) границе для определения плотности газа можно использовать уравнение Бернулли

$$
\rho=\rho_{0}\left(1-\frac{w_{z}^{2}+w_{r}^{2}}{2 w_{s 0}^{2}}\right),
$$

а для безразмерной концентрации примеси в газе ее начальное значение $C_{0}=1$. Для остальных переменных на этой и других открытых границах используются 
те же формулы, что и во внутренних точках, с тем отличием, что для аппроксимации производных в направлении, перпендикулярном к границе, вместо центральных разностей используются односторонние разности „назад“ (или „вперед“) по двум соседним точкам, как, например, на боковой границе при $i=n$

$$
\partial C[n, j] / \partial r \cong(C[n, j]-C[n-1, j]) / h
$$

или на нижней (выходной) границе при $j=n z$

$$
\partial C[i, n z] / \partial z \cong(C[i, n z]-C[i, n z-1]) / h
$$

соответственно.

\section{Результаты расчета массообмена фаз в свободном факеле}

Вышеописанный алгоритм был реализован с использованием программных средств Delphi для расчета межфазного массообмена в двухфазном потоке осесимметричного факела распыла центробежно-струйной форсунки с диаметром сопла $2 \mathrm{~mm}$.

В численном эксперименте для напряжения турбулентного трения газа использовалась зависимость (11), для коэффициента сопротивления капель - формулы (13) и (14).

За единицу безразмерного пространственного масштаба сетки был принят радиус факела на верхней границе расчетной области $r_{0}=z_{0} \operatorname{tg} \varphi\left(\varphi=32.5^{\circ}-\right.$ половина корневого угла факела, $\left.z_{0}=100 \mathrm{~mm}\right)$, в качестве единицы масштаба скоростей была выбрана начальная скорость истечения жидкости из сопла форсунки $u_{0}=0.75\left(2 P_{l} / \rho_{l}\right)^{1 / 2}$. Расчеты проводились для давления воды на форсунке $P_{l}=5 \cdot 10^{5} \mathrm{~Pa}$, при этом измеренный диаметр капель $d=0.14 \mathrm{~mm}$.

В расчетах было принято: безразмерный шаг сетки $\Delta r=\Delta z=1 / 16$, размеры пространственной области $r_{\text {max }}=\max (i) h, z_{\max }-z_{0}=\max (j) h, h=4 \mathrm{~mm}$. Размеры прямоугольной сетки для свободного факела варьировались до $\max (i)=\max (j)=200$ и обеспечивали достаточную аппроксимацию разностной схемы.

Интересовавшее нас в данной задаче (квази)стационарное состояние потока достигалось в результате эволюции нестационарного решения за „сеточное“ время, примерно в 15-20 раз большее характерного времени $t_{s}=\left(z_{\max }-z_{0}\right) / u_{0}$, за которое капли пролетали бы от верхней до нижней границы расчетной области без учета их торможения в газе.

На рис. 2, 3 приведены результаты расчета по предлагаемой модели межфазного массообмена в осесимметричном свободном факеле распыла форсунки.

На рис. 2 представлены аксиальные профили скоростей фаз и концентрации $C[0, j]$ примеси на оси свободного факела без учета и с учетом кризисов сопротивления капель и межфазного массообмена. Очевидно, кризис сопротивления заметно влияет на профили скоростей каждой из фаз: газ движется медленнее, а капли

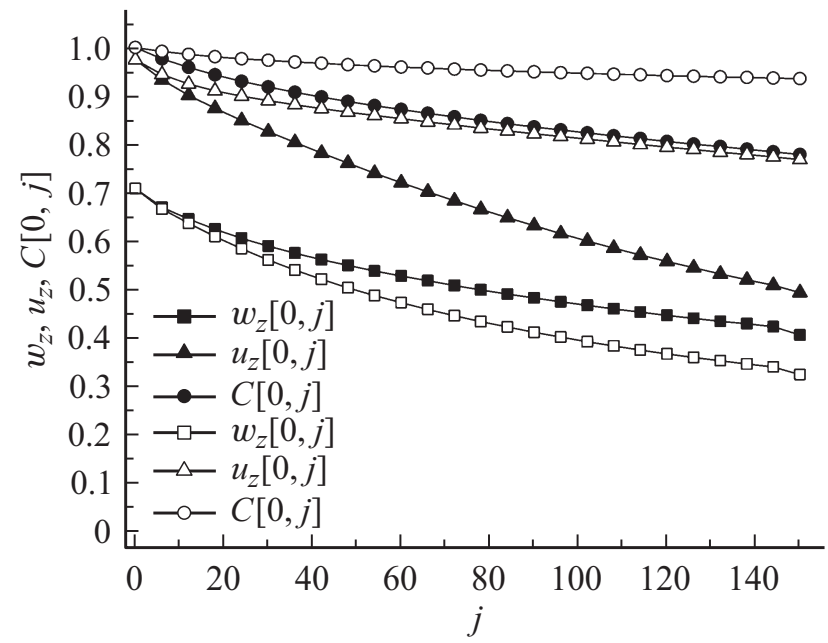

Рис. 2. Расчетные зависимости скоростей газа $w_{z}[0, j]$, капель $u_{z}[0, j]$ и концентрации $C[0, j]$ газовой примеси на оси свободного факела: темные символы - расчет с использованием формул (3) и (17) без учета кризисов сопротивления и массообмена $\left(\mathrm{Nu}_{D}>2\right)$, светлые символы - расчет с учетом кризиса сопротивления по формуле (14) и кризиса массообмена при $\mathrm{Nu}_{D}=2$.

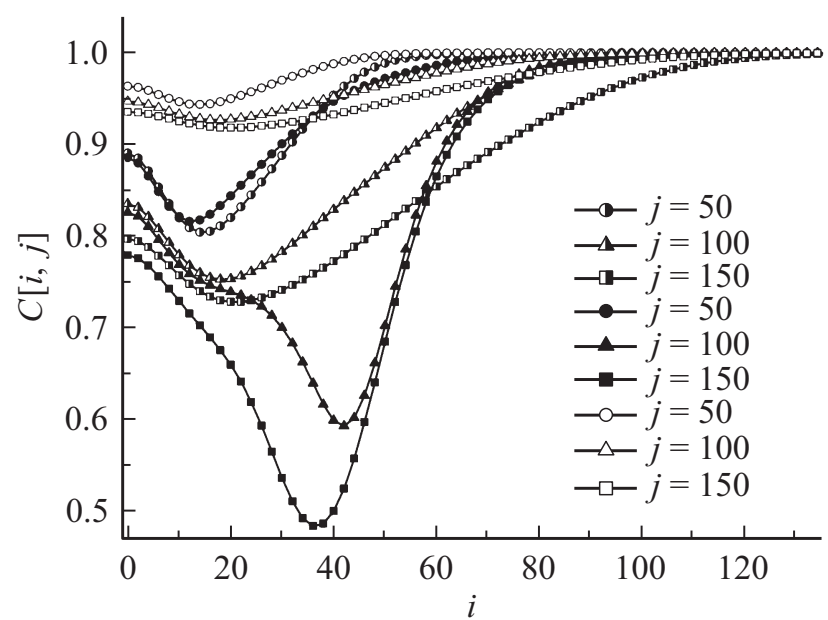

Рис. 3. Расчетные радиальные профили концентрации $C[i, j]$ примеси газа на различных расстояниях $z=(100+4 j) \mathrm{mm}$ от форсунки в свободном факеле распыла: темные символы расчет с использованием формул (3) и (17) без учета кризисов сопротивления и массообмена $\left(\mathrm{Nu}_{D}>2\right)$, светлые символы расчет с учетом кризиса сопротивления по формуле (14) и кризиса массообмена (при $\mathrm{Nu}_{D}=2$ ), наполовину темные и наполовину светлые символы - расчет с учетом кризиса сопротивления, но без учета кризиса массообмена.

быстрее из-за более слабого межфазного обмена импульсом. На межфазный массообмен кризис сопротивления (без учета кризиса массообмена, когда по формуле (17) $\mathrm{Nu}_{D}>2$ ) влияет мало: на расстоянии $z=700 \mathrm{~mm}$ от форсунки (при $j=150$ ) расчетная концентрация примеси с учетом кризиса сопротивления оказалась на $2 \%$ больше, чем без него (на рис. 2 не показано). При 
дополнительном учете кризиса массообмена, когда значения $\mathrm{Nu}_{D}=2$, интенсивность межфазного массообмена значительно снижается, как видно на рис. 2.

На рис. 3 приведены радиальные профили концентрации $C=C[i, j]$ примеси на разных расстояниях $z=100+4 j$ mm от форсунки. Очевидно, кризис сопротивления заметно влияет как на положение минимума концентрации примеси по радиусу факела распыла, так и на ее численное значение в этом минимуме, причем это влияние нарастает с координатой $z$ (при удалении от форсунки). Дополнительный к кризису сопротивления учет кризиса массообмена на положение минимума практически не влияет, но заметно влияет на минимальное и другие значения концентрации примеси в каждом из указанных сечений факела распыла.

\section{Расчет межфазного массообмена в распылительном аппарате}

Используемые в химических технологиях распылительные аппараты условно можно разделить на два типа: инжекторы и эжекторы [14]. В каждом из них имеется форсунка 1 , формирующая факел распыла 2, соосная с форсункой цилиндрическая камера 3 смешения фаз и бак-сепаратор 4 для их разделения (рис. 4). Ось

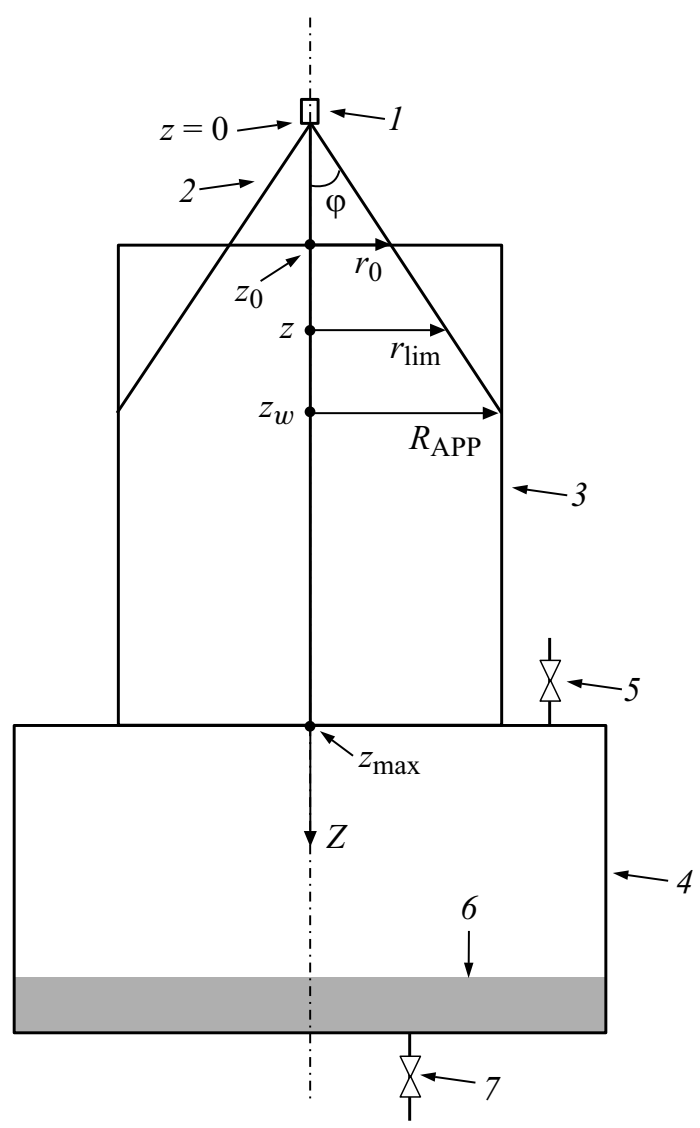

Рис. 4. Схема распылительного аппарата-инжектора для очистки воздуха от газовой примеси, абсорбируемой жидкостью капель. (Пояснения в тексте.)

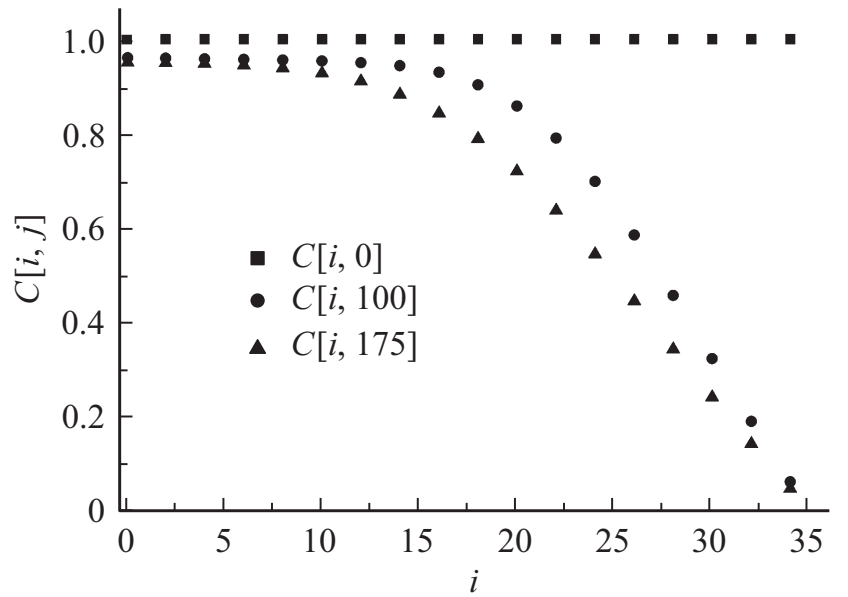

Рис. 5. Расчетные радиальные профили концентрации $C[i, j]$ примеси газа на различных расстояниях $z=(100+4 j) \mathrm{mm}$ от форсунки в аппарате-инжекторе, $R_{\mathrm{APP}}=140 \mathrm{~mm}, H=800 \mathrm{~mm}$.

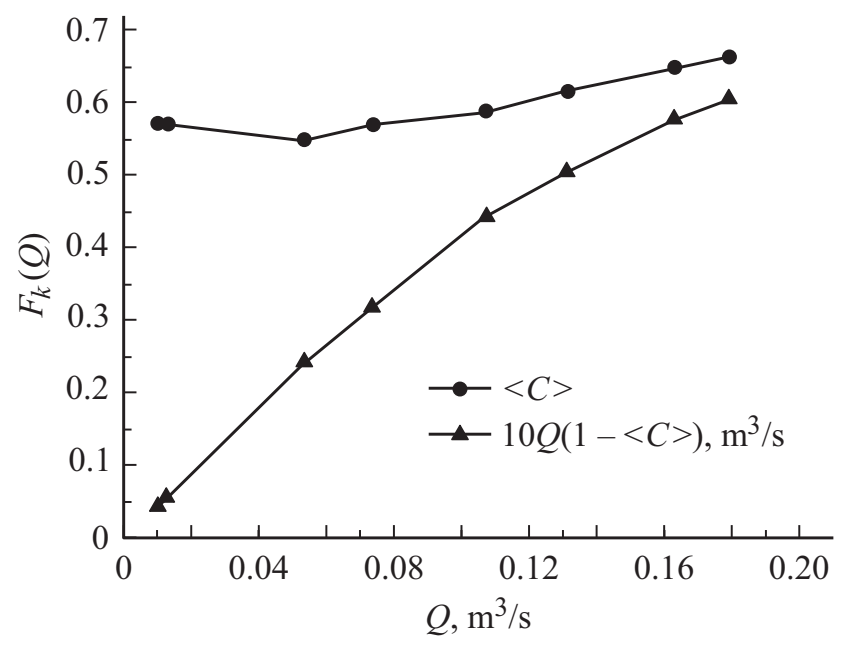

Рис. 6. Расчетные зависимости от ежесекундного объемного расхода $Q$ газа через аппарат для средней (по выходному сечению камеры смешения) концентрации $\langle C\rangle$ примеси и удесятеренного количества $Q(1-\langle C\rangle)$ газовой примеси, поглощенной жидкостью в каплях и в пленке, стекающей по стенке аппарата, $R_{\mathrm{APP}}=140 \mathrm{~mm}, H=1100 \mathrm{~mm}$.

форсунки и камеры - вертикальна. Отличие аппаратов заключается в том, что камера смешения инжектора открыта сверху, расход газа ограничивается вентилем 5 на выходе газа из аппарата. А в эжекторе камера смешения, внутри которой находится форсунка, сверху закрыта, расход газа ограничивается вентилем (на рис. 4 не показан) на входе газа через верхнюю крышку аппарата. Жидкость из слоя 6, накопившегося в бакесепараторе, отводится через вентиль 7.

Цилиндрическая камера смешения фаз ограничивает двухфазный поток по радиусу $\left(r<R_{\mathrm{APP}}\right)$ и высоте $H$, которая связана с положением нижней границы $j=n z$ расчетной области формулой $H=z_{\max }=\left(z_{0}+h n z\right) \mathrm{mm}$. Внутренняя поверхность ее стенки является в рас- 
четной области боковой границей $i=n=R_{\mathrm{APP}} / h$, на которой обе компоненты скорости газа обращаются в нуль, что учитываем, задав условия ,прилипания“: $w_{r}(n, j)=w_{z}(n, j)=0$.

Долетевшие до стенки капли выпадают на нее, смачивая внутреннюю поверхность камеры ниже координаты $z_{w}=R_{\mathrm{APP}} / \operatorname{tg} \varphi$. Для концентрации примесного газового компонента, легко поглощаемого жидкостью капель, испытывались два вида граничных условий на боковой стенке аппарата:

1) $C\left(R_{\mathrm{APP}}, z\right)=1$ при $z<z_{w}$ и $C\left(R_{\mathrm{APP}}, z\right)=0$ при $z \geq z_{w}$

2) $C\left(R_{\mathrm{APP}}, z\right)=0$ при всех возможных значениях $\mathrm{z}$ в предположении, что воздушные вихри могут перенести капли на стенку аппарата выше уровня $z_{w}$.

Результаты расчетов в этих двух случаях отличались незначительно. Но в основной серии расчетов все же использовался первый вариант.

В предлагаемой модели двухфазного потока расчетная область $0<j<n z$ целиком располагается в камере смешения фаз (рис. 4), поэтому алгоритм расчета не зависит от типа распылительного аппарата.

В расчетах задавался перепад давления $\Delta P$ газа между нижним и верхним сечениями камеры смешения границами расчетной области. По рассчитанным значениям аксиальной скорости газа рассчитывался объемный расход газа $Q$ через аппарат. Варианты расчета аппарата отличались перепадом давления $\Delta P$ газа, радиусом $R_{\mathrm{APP}}$ и высотой $H$ камеры смешения инжектора.

На рис. 5 представлены радиальные профили концентрации газовой примеси в аппарате, рассчитанные с учетом обоих кризисов: гидродинамического сопротивления и массопередачи. Очевидно, поглощение газовой примеси вблизи оси аппарата гораздо слабее, чем у его стенки.

На рис. 6 представлены результаты расчета зависимостей для средней (по выходному сечению камеры смешения) концентрации примеси $\langle C\rangle$ и удесятеренного количества $Q(1-\langle C\rangle)$ газовой примеси, поглощенной жидкостью в аппарате, от объемного расхода $Q$ газа через него. Очевидно, средняя концентрация примеси $\langle C\rangle$ немного (примерно на 20\%) возрастает при увеличении расхода газа на порядок. А количество поглощенной жидкостью примеси увеличивается почти так же, как и расход газа.

Слабый рост $\langle C\rangle$ с увеличением $Q$ подтверждает сделанное в работе [10] предположение о том, что максимальная эффективность массообмена в распылительном аппарате будет достигаться при расходе газа, близком к оптимальному $Q_{\text {opt }}$, удовлетворяющему условию

$$
q_{\mathrm{opt}}=Q_{\mathrm{opt}} / Q_{m}=3^{-1 / 2} \text {. }
$$

На рис. 7 показаны расчетные зависимости от площади $S$ поперечного сечения камеры смешения для максимального расхода $Q_{m}$ газа (т.е. при малом перепаде $\Delta P=0.7$ Ра его давления на аппарате), а также величин

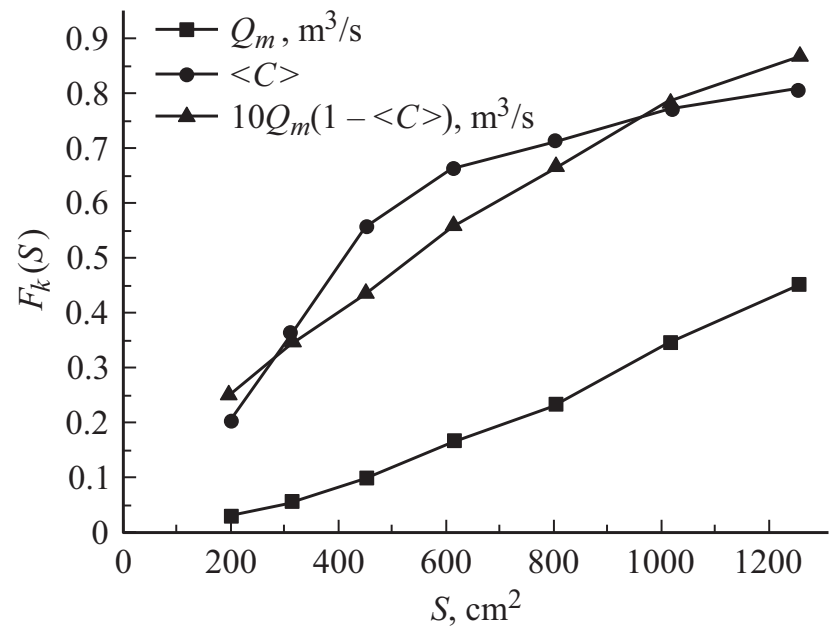

Рис. 7. Расчетные зависимости от площади $S$ поперечного сечения аппарата для максимального расхода $Q_{m}$ газа через аппарат, средней концентрации $\langle C\rangle$ примеси и удесятеренного максимального количества $Q_{m}(1-\langle C\rangle)$ газовой примеси, поглощенной жидкостью, $H=1100 \mathrm{~mm}$, перепад давления газа на аппарате $\Delta P=0.7 \mathrm{~Pa}$.

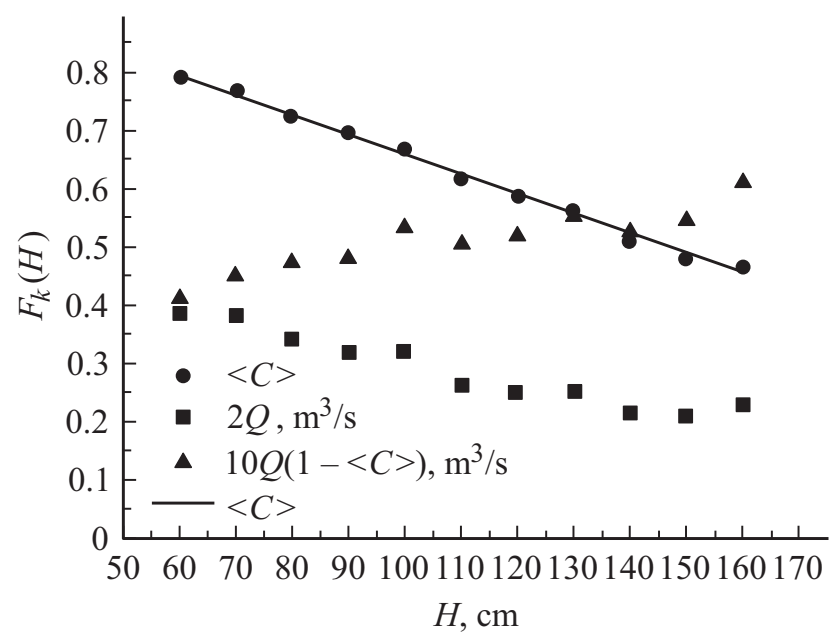

Рис. 8. Расчетные зависимости от высоты $H$ аппарата для средней концентрации примеси $\langle C\rangle$, удвоенного расхода $2 Q$ газа через аппарат и удесятеренного количества $10 Q(1-\langle C\rangle)$ газовой примеси, поглощенной жидкостью, $R_{\mathrm{APP}}=140 \mathrm{~mm}$, перепад давления газа на аппарате $\Delta P=7$ Ра. Сплошной линией показана линейная аппроксимация расчетной зависимости $\langle C\rangle$ от $H$.

$\langle C\rangle$ и $Q_{m}(1-\langle C\rangle)$. Очевидно, все величины возрастают с ростом $S$. Рост $\langle C\rangle$ означает, что интенсивность массообмена фаз и степень очистки воздуха уменьшаются, хотя количество извлеченной из него примеси увеличивается за счет еще большего увеличения расхода $Q_{m}$ газа.

На рис. 8 представлены расчетные зависимости от высоты $H$ аппарата для величин $\langle C\rangle, 2 Q$ и $10 Q(1-\langle C\rangle)$. Очевидно, все величины с ростом $H$ изменяются приблизительно линейно. При увеличении $H$ в 2.7 раза примерно двукратное уменьшение расхода $Q$ газа ком- 
пенсируется почти трехкратным ростом доли $(1-\langle C\rangle)$ поглощенной жидкостью примеси. В результате полное количество поглощенной примеси $Q(1-\langle C\rangle)$ растет в 1.5 раза.

\section{Заключение}

Предложенная ранее в работах $[5,10]$ модель факела распыла форсунки с учетом раннего кризиса сопротивления капель развита в настоящей работе путем учета кризиса массопередачи от газа к каплям, аналогичного кризису теплообмена [12].

По сравнению с предыдущими работами автоpa $[10,12]$, в настоящей работе получены новые результаты. В частности, в свободном факеле распыла высотой $H$ до $0.7 \mathrm{~m}$ рассчитаны не только аксиальные и радиальные профили скоростей фаз, но и распределение концентрации $C(r, z)$ газовой примеси в потоке газа с учетом и без учета обоих кризисов: сопротивления капель и массопередачи.

Кроме свободного факела распыла, межфазный массообмен был рассчитан также в газокапельном потоке через цилиндрический аппарат. Установлены зависимости концентрации $\langle C\rangle$ газовой примеси на выходе аппарата, количества $Q(1-\langle C\rangle)$ поглощенной жидкостью газовой примеси от ежесекундного объемного расхода $Q$ газа через аппарат, а также зависимости трех указанных величин от площади $S$ поперечного сечения и высоты $H$ аппарата.

Представленная численная модель позволяет рассчитать зависимости режимных характеристик $Q,\langle C\rangle$, $Q(1-\langle C\rangle)$ распылительного аппарата от его конструктивных параметров $S$ и $H$ и перепада давления $\Delta P$ газа на нем.

Согласно рис. 6-8, из-за кризиса массопередачи степень очистки газа от примеси $(1-\langle C\rangle)$ невелика не превышает 0.5. Поэтому стоит рассмотреть возможность использования нескольких ступеней очистки воздуха от примеси.

\section{Конфликт интересов}

Автор заявляет, что у него нет конфликта интересов.

\section{Список литературы}

[1] Cmpayc B. Промышленная очистка газов. М.: Химия, 1981. $616 \mathrm{c}$

[2] Нигматулин Р.И. Динамика многофазных систем. Ч. 1. М.: Наука, 1987. 464 с.

[3] Абрамович Г.Н. Теория турбулентных струй. М.: Наука, 1984. $716 \mathrm{c}$.

[4] Симаков Н.Н. // ЖТФ. 2004. Т. 74. Вып. 2. С. 46-51. [Simakov N.N. // Tech. Phys. 2004. Vol. 49. N 2. P. 188-193.]

[5] Simakov N.N., Simakov A.N. // J. Appl. Phys. 2005. Vol. 97. P. 114901.
[6] Torobin L.B., Gauvin W.H. // Can. J. Chem. Eng. 1959. Vol. 37. N 4. P. 129-141.

[7] Шлихтинг Г. Теория пограничного слоя / Пер. с нем. М.: Наука, 1974. 712 с.

[8] Симаков Н.Н. // ЖТФ. 2010. Т. 80. Вып. 7. С. 1-7. [Simakov N.N. // Tech. Phys. 2010. Vol. 55. N 7. P. 913-919. DOI: $10.1134 / \mathrm{S} 1063784210070017]$

[9] Симаков Н.Н. // ЖТФ. 2013. Т. 83. Вып. 4. С. 16-20. [Simakov N.N. // Tech. Phys. 2013. Vol. 58. N 4. P. 481-485. DOI: $10.1134 / \mathrm{S} 1063784213040233]$

[10] Симаков Н.Н. // ЖТФ. 2017. Т. 87. Вып. 7. С. 990-996. DOI: 10.21883/0000000000 [Simakov N.N. // Tech. Phys. 2017. Vol. 62. N 7. P. 1006-1012. DOI: $10.1134 / \mathrm{S} 1063784217070222]$

[11] Лойиянский Л.Г. Механика жидкости и газа. М.: Наука, 1978. $736 \mathrm{c}$.

[12] Симаков Н.Н. // ЖТФ. 2016. Т. 86. Вып. 12. С. $42-48$. [Simakov N.N. // Tech. Phys. 2016. Vol. 61. N 12. P. 18061812. DOI: 10.1134/S1063784216120252]

[13] Поттер Д. Вычислительные методы в физике / Пер. с англ. М.: Мир, 1975. 392 с.

[14] Автореф. докт. дис. Симаков Н.Н. Ярославль: ЯГТУ, 2003. $354 \mathrm{c}$. 